\title{
Analysis of internal environment
}

\author{
Mirela Dogaru \\ Faculty of Marketing \\ "Dimitrie Cantemir" Christian University \\ E-mail: mireladogaru77@yahoo.com \\ Robert Chira \\ Faculty of Marketing \\ "Dimitrie Cantemir" Christian University
}

\begin{abstract}
Company performance Druckfarben Hellas is influenced by the ability to integrate into their environment, the effectiveness of its actions in the opportunities, the ability to cope with adverse situations and risks facing. Assessment methods used are SWOT analysis, identifying critical success factors and profile capability. Resources are fall in production or services and are achieving individual contributions, social or organizational. Keywords: Company performance, SWOT resources.
\end{abstract}

Company performance Druckfarben Hellas is influenced by the ability to integrate into their environment the effectiveness of its actions in the opportunities, the ability to cope with adverse situations and risks facing.

In order to adopt an appropriate behavior in relation to its environment, Druckfarben Hellas Company considers the following elements:

- complex nature of the environment;

- the status of the system environment and the fact that it is made up of a set of subsystems:

O a micro environment - is the factor that directly affects the company's ability to achieve its goals;

a macro environment - is the factor that affects the activity of all components of the microenvironment;

o a global environment - Analysis International / global.

Environment Druckfarben Hellas company consists of a set of constraints against which society must adapt, choosing certain means to this end. Based on information about the environment, she chooses a certain strategy to meet objectives and a specific structure which enable the implementation strategy.

In relation to the company, Druckfarben Hellas adopt for two types of behavior: 
- one adaptive, reacting to the environment because it is dependent on the resources that provide them;

- proactive, with the ability to act on the environment induces some changes. The selection of certain options is determined by the way she perceives the environment in which it operates and its resources.

The main task of management is to provide information service, performance and adapt to internal and external change. The initial information from internal and external environment must be collected, treated and evaluated.

Assessment methods used are SWOT analysis, identifying critical success factors and profile capability.

Slack management would be to ignore or overlook the ongoing review of the performance of the organization against internal and external environment. Often, such an excuse for the practice is that the time consuming. Those who make such statements should reflect the advantages revision to reduce risk factors in decision making and for providing other opportunities. At the same time, minimizing threats and weaknesses, planning becomes more efficient through the use of such a process.

The purpose of the analysis of internal in the company is to present its structure and importance of a careful analysis of the external environment in order to obtain strategic advantages. After studying them, will be able to answer the following questions:

- the importance of studying and understanding the internal environment of the organization;

- tangible and intangible resources of the organization;

- the difference between tangible and intangible resources;

- the organization's capabilities and explanations of how they can grow;

- Fundamental skills and explanations to obtain strategic advantages;

- how value chain analysis;

- Icarus paradox and inertia success.

\section{Resources needed}

To develop and implement strategies to ensure success of the organization is necessary to analyze the internal and coupling the results with those obtained in the analysis of the external environment. By studying the external environment, the organization identifies what could be done, given the competitive context and external environment generally favorable or adverse.

By studying the internal environment, Druckfarben Hellas determine what they can do actually. By coupling the two conclusions can decide which strategy to develop the organization to ensure sustainable strategic competitiveness. 
Robert Grant considers internal environmental analysis even more important: "While the external environment is constantly changing, their resources and capabilities of the organization are defined in relation to a more stable environment". [Robert Grant-1991]

Resources are items fall in production or services and are achieving individual contributions, social or organizational. Resources can not ensure the success of the organization materialize their mere presence as a competitive advantage. The company may have sufficient material resources and equipment but if you have good management, efficiency and effectiveness of their use may be unsatisfactory. But if there is a well established team spirit and a clear strategy on the field of victory is uncertain. Resources are important, but only their integration in a system performance can lead to competitive advantages.

Resources of the organization are grouped Druckfarben Hellas tangible resources and intangible resources. Tangible resources are those resources that can be seen and quantified. Intangible resources are more difficult to define, but they take different forms of intellectual property, knowledge and know- how. An important intangible resource is the organization's reputation, which can be exploited intelligently when it is synonymous with quality and performance.

Tangible resources can be grouped as follows:

- Financial resources: Financial lending capacity of the organization, the ability to generate their own revenue and financial inflows generated by the organization itself;

- Natural resources: equipment performance, efficient production technologies, factories and infrastructure, land valuable as the size, quality and location;

- Human resources: employees in executive and managerial positions to be distinguished by intelligence, creativity, experience, general and specialized studies, loyalty and adaptability;

- Organizational resources: the functional structure of the organization, effectiveness connections, communication, coordination and control, respectively.

Intangible resources can be grouped into:

- technological resources stock patents and trademarks, intellectual property and industrial secrets. Additionally, add the knowledge to apply them correctly and effectively;

- resources for innovation: an institutional culture based on models of creative thinking, research laboratories, specialized documentation to enable innovation and an attitude of acceptance of risk;

- reputation: the reputation of the organization in relation to the beneficiaries or her customers perception of quality products, durable and reliable reputation of the organization in relation to suppliers resulting from a collaboration efficient and reliable.

Tangible and intangible resources are sources of the organization and capabilities in the development of basic skills, competitive advantages. 
Intangible resources within the company have a high potential for generating basic skills, demonstrated superior potential of tangible resources. Recent experience has shown that the company's success was assured a greater intellectual capacity and organizational effectiveness than the existence of primary materials, equipment and buildings.

Ability to ensure effective management of human intelligence and its transformation products and quality services has become increasingly present and future requirement. A number of statistics shows that the share of intangible resources in total resources company growing. There is a very simple situation: intangible resources are less visible and more difficult to understand for competitors to be imitated, duplicated or substituted.

\section{Capabilities}

Capability of Drukfarben Hellas company in the chemical industry reflects its ability to use resources more efficiently, so that the existing potential, becomes beneficial results for the organization as much.

Organization Druckfarben Hellas aebe thus characterized by the existence of resources and capabilities and differentiates itself from other organizations the ability to exploit them in a competitive environment. Capabilities are intangible in nature and are sometimes harder to define or evaluate but they can be clearly identified by the results produced. For good management is very important distinction between resources and capabilities. The company has valuable resources, but if you have the necessary capabilities to use as clever and creative these resources, then it can not gain a competitive advantage in the external environment.

Capabilities are obtained by time integration of the quality of human resources, knowledge, organizational structure and organizational culture. To better understand this, we will consider some examples:

- $\quad$ In the distribution of products is an important capability in logistics developed and used by the firm;

- Human resources can be highlighted company's ability to motivate employees to build solutions as creative, as happens in the company Druckfarben Hellas aebe

The management of this company has always placed great emphasis on developing teamwork employees.

A growing emerging capability is the ability to be a learning organization. This means continuous development and improvement of the knowledge base of knowledge management. Also important is the rate of acquisition of new knowledge and integration into the existing structure. Druckfarben Hellas even set up station chief learning officer (CLO), and senior managers in charge of the learning organization.

\section{Core Skills}


Having the necessary knowledge resources and capabilities of the organization, senior management is prepared to identify and develop basic skills, ie build support for achieving strategic advantage in competitive external environment. Fundamental skills contribute to the organization's personality and allow them to differentiate themselves favorably to other organizations in competitive external environment. Integrating resources and capabilities of an organization in a creative and effective way, we obtain the fundamental skills that are unique to the organization and enhance value for its clients for a period of time that is meant to be as long.

We must point out that not every company resources and capabilities can be integrated to generate fundamental skills. While any key competence is based on capability, not every capability is transformed automatically into a fundamental skill. It may consider the following criteria to see whether the organization capabilities Druckfarben Hellas can turn into fundamental skills: value, rarity, cost of imitation and substitution opportunities.

Capabilities company values that create value for the firm by exploiting opportunities and neutralize threats in the external environment. These capabilities allow company management to formulate and implement strategies that create value for certain beneficiaries.

Rare are those capabilities that are present in very few of the current or potential competitors. Assessing the capabilities of the organization's managers must ask and answer to many other organizations can identify these capabilities is to decide whether they can be considered rare or not. Capabilities that are found in Druckfarben Hellas and are engaged in the same competition competitive advantage can not be support for any of them. Competitive advantage can occur only when the organization develops a certain capability that is unique or is found in very few competitors.

Capabilities that are costly to imitate contribute significantly to building competitive advantage. These capabilities can be developed as a result of unique historical conditions. One can illustrate this case Druckfarben Hellas company that has developed from the beginning a culture of innovation and excellence.

There are situations where it is difficult to make a direct link between competitive advantage and capabilities that were obtained at base. Uncertainty that makes it difficult to identify successful generating capability contributes to the increased cost of any imitation of those capabilities by competitors. There may be some social complexity make it difficult to imitate successfully generating capability. Organizational culture of the company Druckfarben Hellas is very complex and difficult to imitate to get the same competitive advantage.

Capabilities that may be substituted are those which have an equivalent strategy. The strategic value of a capability increases with decreasing her chances of substitution by competing organizations. Specific expertise Druckfarben Hellas and the existence of trust and cooperation between managers and staff can be capabilities that can not be substituted by any other competitors, in order to achieve competitive advantage. 
Once created, competitive advantage should be maintained as much to achieve strategic competitiveness. The success of such a strategy depends on three factors: the creation of barriers to limit imitation of basic skills, capabilities available to competitors and the general dynamics of the external environment, especially competitive environment.

When the organization achieves a competitive advantage, other organizations that are competing will try to identify the resources and capabilities that led to the success and emulate. The question is how long will achieve this. Speed limit contributes to the erosion of competitive advantage. Therefore, the organization conducting the competitive advantage they need to invest continuously to be one step ahead of your competitors. This means to create new barriers for new competitors to reduce the chances of imitation and, respectively, to increase the time available to the organization that has achieved competitive advantage. Time becomes a crucial element of competition.

When competitive advantage is based on particular resources, their imitation can be done relatively quickly because they are easily identified and replicated. Intangible resources are more difficult to identify and thus they become more difficult to imitate.

An important barrier that can be placed in the path of imitation is to create a company name, as did Druckfarben Hellas and more. This name is linked to resources, capabilities and some know-how which is the true secret of the company. Imitation is more difficult than the capabilities of resources because they are not so obvious and explicit.

\section{References}

1. Peterson, N.G., Mumford, M.D. Borman, W.C. et al. ,2001, Under- standing work using the occupational information network (ONET): Implications for practice and research, Personnel Psychology, 2001.

2. Henry, J., 2001, Creative management, Sage, London.

3. Huczynski A., Buchanan D., 2001, Organizational Behaviour. An Introductory Text, 4th Edition Prentice Hall, 2001.

4. Bass, B., 1990, Boss \& Stogdill's handbook ofleadership: Theory, research and managerial applications, Free Press, New York.

5. Anderson, N.R., Thomas, 1996, H.D.C. Work group socialization, in M.A. West (editor), The handbook of work group psychology, Wiley, Chichester, UK. 